\title{
High WT1 expression is an early predictor for relapse in patients with acute promyelocytic leukemia in first remission with negative PML-RARa after anthracycline-based chemotherapy: a single-center cohort study
}

Jae-Ho Yoon, Hee-Je Kim", Dae-Hun Kwak, Sung-Soo Park, Young-Woo Jeon, Sung-Eun Lee, Byung-Sik Cho, Ki-Seong Eom, Yoo-Jin Kim, Seok Lee, Chang-Ki Min, Seok-Goo Cho, Dong-Wook Kim, Jong Wook Lee and Woo-Sung Min

\begin{abstract}
Wilms' tumor gene 1 (WT1) expression is a well-known predictor for relapse in acute myeloid leukemia. We monitored WT1 decrement along the treatment course to identify its significant role as a marker for residual disease in acute promyelocytic leukemia (APL) and tried to suggest its significance for relapse prediction. In this single center retrospective study, we serially measured PML-RARa and WT1 expression from 117 APL patients at diagnosis, at post-induction and post-consolidation chemotherapies, and at every 3 months after starting maintenance therapy. All 117 patients were in molecular remission after treatment of at least 2 consolidation chemotherapies. We used WT1 ProfileQuant ${ }^{\text {TM }}$ kit (Ipsogen) for WT1 monitoring. High WT1 expression (>120 copies $/ 10^{4}$ ABL1) after consolidation and at early period (3 months) after maintenance therapy significantly predicted subsequent relapse. All paired PML-RARa RQ-PCR were not detected except for one sample with early relapse. Patients with high WT1 expression at 3 months after maintenance therapy $(n=40)$ showed a significantly higher relapse rate (30.5 vs. 6.9\%, $P<0.001)$ and inferior disease free survival ( 62.8 vs. $91.4 \%, P<0.001)$. Multivariate analysis revealed that high peak leukocyte counts at diagnosis $(H R=6.4, P<0.001)$ and high WT1 expression at 3 months after maintenance therapy $(H R=7.1, P<0.001)$ were significant factors for prediction of relapse. Our data showed high post-remission WT1 expression was a reliable marker for prediction of subsequent molecular relapse in APL. In this high-risk group, early intervention with ATRA \pm ATO, anti-CD33 antibody therapy, and WT1-specific therapy may be used for relapse prevention.
\end{abstract}

Trial registration: Clinical Research Information Service (CRIS), KCT0002079

Keywords: Acute promyelocytic leukemia, WT1, FLT3 mutation, Minimal residual disease

\footnotetext{
* Correspondence: cumckim@catholic.ac.kr

Department of Hematology, Catholic Blood and Marrow Transplantation Center, Leukemia Research Institute, Seoul St. Mary's Hospital, College of Medicine, The Catholic University of Korea, 222 Banpodaero, Seocho-gu, Seoul 06591, Korea
} 
Table 1 Baseline characteristics of enrolled patients

\begin{tabular}{|c|c|}
\hline Total $n=117$ & Number or median value \\
\hline Age, median (range) & $44(19-70)$ \\
\hline Gender, male & $70(59.8 \%)$ \\
\hline \multicolumn{2}{|l|}{ Laboratory findings at diagnosis } \\
\hline Leukocyte count $\left(\times 10^{9} / \mathrm{L}\right)$ & $2.68(0.4-112.0)$ \\
\hline Leukocytes count at peak $\left(\times 10^{9} / \mathrm{L}\right)$ & $16.6(0.4-112.0)$ \\
\hline Hemoglobin (g/dL) & $8.9(4.0-15.0)$ \\
\hline Platelet $\left(\times 10^{9} / \mathrm{L}\right)$ & $33.0(5.0-163.0)$ \\
\hline Lactate dehydrogenase (LDH, U/L) & $692(250-4070)$ \\
\hline Prothrombin time (PT, \%) & $63.0(35.0-101.0 \%)$ \\
\hline Partial thromboplastin time (aPTT, s) & $28.0(20-45)$ \\
\hline Fibrinogen (mg/dL) & $134.0(31.0-500.0)$ \\
\hline Antithrombin III (\%) & $94.0(49.0-150.0)$ \\
\hline D-dimer (mg/L) & $17.0(1.0-36.0)$ \\
\hline \multicolumn{2}{|l|}{ Sanz criteria } \\
\hline High & $64(54.7 \%)$ \\
\hline Intermediate & $19(16.2 \%)$ \\
\hline Low & $34(29.1 \%)$ \\
\hline \multicolumn{2}{|l|}{ Karyotype } \\
\hline Normal karyotype & $5(4.3 \%)$ \\
\hline$t(15 ; 17)$ alone & $79(67.5 \%)$ \\
\hline $\mathrm{t}(15 ; 17)$ with 1 additional karyotype & $20(17.1 \%)$ \\
\hline $\mathrm{t}(15 ; 17)$ with $\geq 2$ additional karyotype & $13(11.1 \%)$ \\
\hline \multicolumn{2}{|l|}{ PML-RARa subtype } \\
\hline BCR1 & $85(72.6 \%)$ \\
\hline BCR3 & $32(27.4 \%)$ \\
\hline \multicolumn{2}{|l|}{ FLT3 mutation } \\
\hline No FLT3 mutation & $86(73.5 \%)$ \\
\hline FLT3-ITD & $25(21.4 \%)$ \\
\hline FLT3-TKD & $6(5.1 \%)$ \\
\hline \multicolumn{2}{|l|}{ WT1 (copies/104ABL), median (range) } \\
\hline At diagnosis $(n=117)$ & $18330(20.0-236160.0)$ \\
\hline Post-induction ( $n=117)$ & $63.9(4.9-2360.0)$ \\
\hline Post 1st consolidation $(n=117)$ & $66.2(1.1-2320.0)$ \\
\hline Post 2nd consolidation ( $n=117$ ) & $80.1(1.3-2110.0)$ \\
\hline Post 3rd consolidation ( $n=117$ ) & $71.9(10.8-808.0)$ \\
\hline aPost-maintenance 3 months ( $n=117$ ) & $70.0(6.0-5520.0)$ \\
\hline a Post-maintenance 1 year $(n=87)$ & $57.5(10.0-1630.0)$ \\
\hline 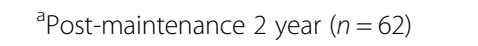 & $54.4(10.0-500.0)$ \\
\hline At relapse $(n=16)$ & $239.5(77.1-34910.0)$ \\
\hline Leukapheresis at initial treatment & $20(17.1 \%)$ \\
\hline Differentiation syndrome & $21(17.9 \%)$ \\
\hline \multicolumn{2}{|l|}{ Hematological complete response } \\
\hline After induction & $115(98.3 \%)$ \\
\hline After 2nd induction & $2(1.7 \%)$ \\
\hline
\end{tabular}

Table 1 Baseline characteristics of enrolled patients (Continued)

\begin{tabular}{|c|c|}
\hline \\
\hline \multicolumn{2}{|c|}{$\begin{array}{l}\text { Complete molecular response (CMR) } \\
\text { After induction }\end{array}$} \\
\hline After 2nd induction & $2(1.7 \%)$ \\
\hline After 1st consolidation & 42 (35.9\%) \\
\hline After 2nd consolidation & $5(4.3 \%)$ \\
\hline
\end{tabular}

\section{Findings}

In acute promyelocytic leukemia (APL), PML-RARa transcript is used as a marker for minimal residual disease (MRD), but the marker is not useful for pre-emptive management since its positivity directly indicates relapse. High Wilms' tumor gene 1 (WT1) expression was related with subsequent relapse in acute myeloid leukemia, and Hecht et al. recently reported that high initial WT1 expression was associated with more relapse in APL [1-3].

We confirmed APL by chromosome analysis and PML$R A R \alpha$ reverse transcriptase polymerase chain reaction (RT-PCR) method. All were treated with idarubicin (12 $\mathrm{mg} / \mathrm{m}^{2}$, days $1,3,5$, and 7$)$ and all-trans retinoic acid (ATRA; $45 \mathrm{mg} / \mathrm{m}^{2} /$ day) $[4,5]$. After achievement of hematological complete remission (CR), all received three courses of consolidation-first, idarubicin $\left(7 \mathrm{mg} / \mathrm{m}^{2}\right.$, days $1-4)$; second, mitoxantrone $\left(10 \mathrm{mg} / \mathrm{m}^{2}\right.$, days $\left.1-4\right)$; and third, idarubicin $\left(12 \mathrm{mg} / \mathrm{m}^{2}\right.$, day $\left.1-2\right)$-followed by 2-year maintenance using 6-mercaptopurine $\left(50 \mathrm{mg} / \mathrm{m}^{2} /\right.$ day) plus ATRA [5-7]. The molecular studies were performed at diagnosis and 1 month after chemotherapy, and every 3 months after maintenance. Quantification of PML-RAR $\alpha$ and WT1 were performed using the realtime quantitative (RQ)-PCR methods (Real-Q PML$R A R \alpha$ quantification kit, Biosewoom, Korea, and WT1 ProfileQuant $^{\text {ti }}$ kit, Ipsogen, France) presenting a similar sensitivity of $4.5 \mathrm{log}$.

We initially identified 142 APL patients from 2009 to 2014 but finally focused on 117 patients (median age 44 years old (range 19-70 years)) who underwent at least 2 cycles of consolidation after hematological CR. All patients were in complete molecular response (CMR) at the time of enrollment (Additional file 1: Figure S1, Table 1). Relapse was identified in 16 (13.7\%) patients with a median duration of 22.8 months (range, 4.3-64.0). After median follow-up of 46.0 months (range, 14.7-86.3), 4year cumulative incidence of relapse (CIR), non-relapse mortality (NRM), disease-free survival (DFS), and overall survival (OS) rates were 16.2, 1.2, 82.6, and 92.5\%, respectively. We identified that high-risk Sanz-criteria, peak leukocyte count $>40.0 \times 10^{9} / \mathrm{L}$, and FLT3 mutation were predictive for relapse. 
We compared the level of WT1 between relapsed and non-relapsed group during the course of treatment (Additional file 1: Figure S2) and identified that median WT1 was significantly different at post 2 nd consolidation (171.5 vs. $76.3, P=0.049$ ), at post 3 rd consolidation (156.0 vs. $67.6, P=0.013)$ and at 3 months postmaintenance (162.0 vs. $59.1, P=0.002)$. We found that WT1 post-maintenance 3 months was the most significant parameter for relapse prediction at the cutoff of $\geq 120.0$ copies $/ 10^{4} A B L$.

We calculated subsequent CIR and DFS rates in 116 patients after excluding 1 patient with early relapse. Patients with WT1 post-maintenance 3 months higher than 120.0 copies $/ 10^{4} A B L$ showed higher 4 -year CIR (30.5 vs. $6.9 \%$,
$P=0.0002)$ and inferior 4 -year DFS (62.8 vs. $91.4 \%, P<$ 0.0001 ) rates (Fig. 1a, b). Also in the high-risk subgroup, high WT1 post-maintenance 3 months showed higher 4-year CIR (43.3 vs. $11.1 \%, P<0.0001)$ and inferior 4-year DFS (55.5 vs. $86.4 \%, P=0.0015)$ rates (Fig. 1c, d). In FLT3 positive and negative subgroup, high WT1 post-maintenance 3 months showed higher 4-year CIR (51.4 vs. $0.0 \%, P<0.0001$ and 21.5 vs. $8.6 \%, P=0.0434$ ) and inferior 4-year DFS (46.7 vs. $100.0 \%, P=0.0018$ and 69.6 vs. $89.3 \%, P=0.0154$ ) rates (Fig. 1 e, $f$ ).

Multivariate analysis (Additional file 1: Table S1) revealed that 4-year CIR was significantly higher in patients with high peak leukocyte count $(\mathrm{HR}=6.414 ; 95 \% \mathrm{CI}, 2.1-19.3$, $P<0.001)$ and high $W T 1$ post-maintenance 3 month

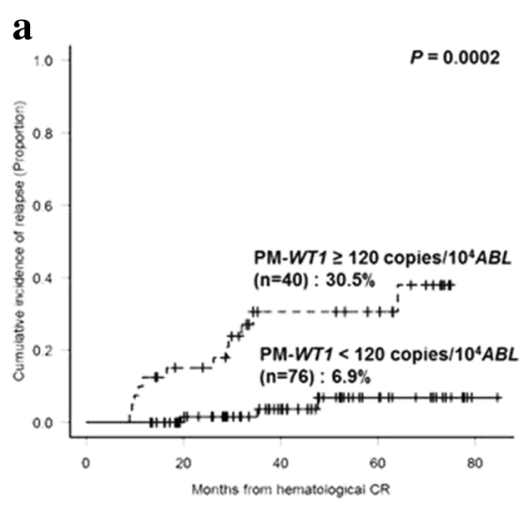

C
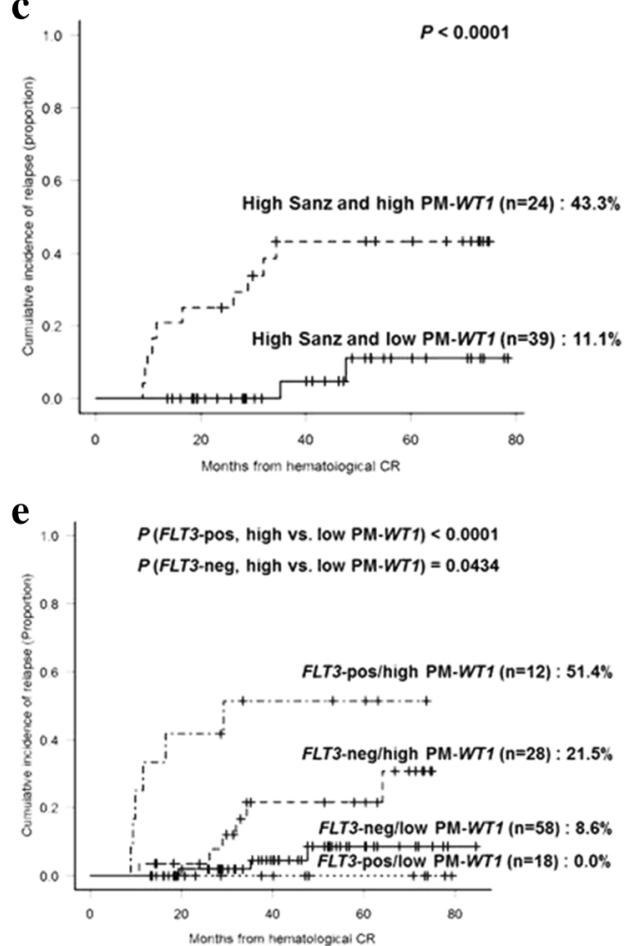

b

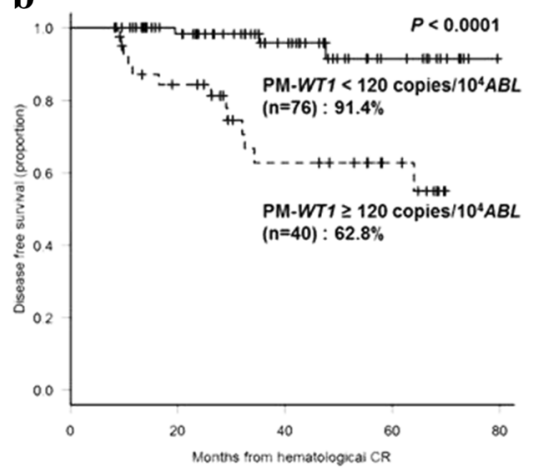

d

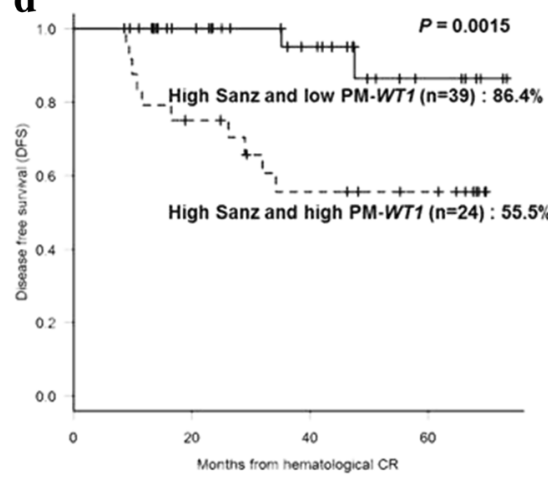

$\mathbf{f}$

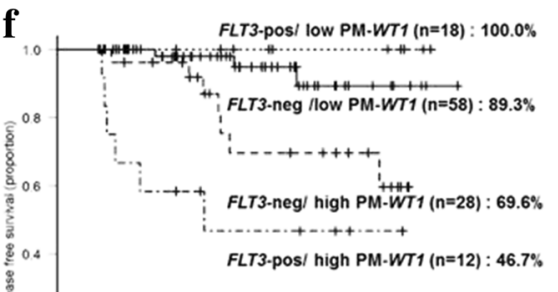

$P($ FLT3-pos, high vs. low PM-WT1 $)=0.0018$ $P($ FLT3-neg, high vs. low PM-WT1) $=0.0154$ Montris trom nematoogical $\quad 60$

Fig. 1 Treatment outcomes according to WT1 expression level (<120 vs. $\geq 120$ copies $/ 10^{4} \mathrm{ABL}$ ) at 3 months post-maintenance (PM-WT1). a Four-year CIR rates. b Four-year DFS rates. $\mathbf{c}$, d Four-year CIR and DFS rates according to WT1 expression level in the high-risk subgroup. e, $\mathbf{f}$ Four-year CIR and DFS rates according to the status of PM-WT1 and FLT3-ITD mutation 
( $\mathrm{HR}=7.533 ; 95 \% \mathrm{CI}, 2.3-24.8, P<0.001)$, and 4 -year DFS was significantly inferior in patients with high peak leukocyte count $(\mathrm{HR}=5.275$; 95\% CI, 1.9-14.7, $P=0.001$ ) and high WT1 post-maintenance 3 month $(\mathrm{HR}=8.241$; 95\% CI, 2.3-29.1, $P=0.001$ ).

Unfortunately, our chemotherapy regimen was not differently specified for high-risk APL and the standard treatment of APL is now changed to a combination therapy using ATO. Therefore, current results may not be applicable in the treatment course using ATO and another validation is needed. Conclusively, high post-remission WT1 expression is a reliable marker for prediction of subsequent relapse in APL patients treated with conventional chemotherapy. For patients with high-risk of relapse, early intervention using WT1-specific therapy may prevent relapse and improve survival outcomes $[8,9]$.

\section{Additional file}

Additional file 1: Table S1. Multivariate analysis in APL patients with CMR. Figure S1. Consort diagram of enrolled patients in this study. Underlined patients were excluded in this study $(n=25)$. Abbreviation: $A P L$, acute promyelocytic leukemia; ATRA, all-trans retinoic acid; CR, complete remission, CMR, complete molecular response; WT1, Wilms tumor 1. Figure S2. Comparison of PML-RARa and WT1 expression levels between relapsed and non-relapsed patients from diagnosis to relapse or 1 year after starting maintenance for non-relapsed patients. (DOCX 152 kb)

\section{Acknowledgements}

Not applicable.

\section{Funding}

This study was supported by the Research Fund of Seoul St. Mary's Hospital, The Catholic University of Korea, and also supported by a grant from the Basic Science Research Program through the National Research Foundation of Korea (NRF) funded by the Ministry of Education (2015R1D1A1A01059819).

\section{Availability of data and materials}

The data of the current study are available from the corresponding author on a reasonable request.

\begin{abstract}
Authors' contributions
J-HY performed the molecular research, collected and analyzed data, and wrote the manuscript. D-HK, S-SP, B-SC, Y-WJ, S-EL, K-SE, Y-JK, SL, C-KM, S-GC, $D-W K, J W L$, and W-SM provided patients and materials and reviewed the manuscript. H-JK designed and conducted the study, provided patients and materials, analyzed data, and wrote the manuscript. All authors read and approved the final manuscript.
\end{abstract}

\section{Competing interests}

The authors declare that they have no competing interests.

\section{Consent for publication}

The consent for publication is not applicable for this study and is permitted by the Institutional Review Board and Ethics Committee guidelines of the Catholic Medical Center (KC15RISI0862).

\section{Ethics approval and consent to participate}

This research was conducted in accordance with the Institutional Review Board and Ethics Committee guidelines of the Catholic Medical Center (KC15RISI0862). Additionally, this research is also permitted and registered in Clinical Research Information Service (CRIS) which is connected to WHO ICTRP; Korea Centers for Disease Control and Prevention, Ministry of Health and Welfare (Republic of Korea); KCT0002079.
Received: 17 November 2016 Accepted: 18 January 2017

Published online: 23 January 2017

\section{References}

1. Hecht A, Nowak D, Nowak V, et al. A molecular risk score integrating BAALC, ERG and WT1 expression levels for risk stratification in acute promyelocytic leukemia. Leuk Res. 2015.

2. Hecht A, Nolte F, Nowak D, et al. Prognostic importance of expression of the Wilms' tumor 1 gene in newly diagnosed acute promyelocytic leukemia. Leuk Lymphoma. 2015;56(8):2289-95.

3. Yoon JH, Kim HJ, Jeon YW, et al. Outcome of allogeneic hematopoietic stem cell transplantation for cytogenetically normal AML and identification of high-risk subgroup using WT1 expression in association with NPM1 and FLT3-ITD mutations. Genes Chromosomes Cancer. 2015.

4. Sanz MA, Martin G, Rayon C, et al. A modified AIDA protocol with anthracycline-based consolidation results in high antileukemic efficacy and reduced toxicity in newly diagnosed PML/RARalpha-positive acute promyelocytic leukemia. PETHEMA group. Blood. 1999;94(9):3015-21.

5. Lee S, Kim YJ, Eom KS, et al. The significance of minimal residual disease kinetics in adults with newly diagnosed PML-RARalpha-positive acute promyelocytic leukemia: results of a prospective trial. Haematologica. 2006;91(5):671-4.

6. Sanz MA, Martin G, Gonzalez M, et al. Risk-adapted treatment of acute promyelocytic leukemia with all-trans-retinoic acid and anthracycline monochemotherapy: a multicenter study by the PETHEMA group. Blood. 2004;103(4):1237-43.

7. Sanz MA, Montesinos P, Vellenga $E$, et al. Risk-adapted treatment of acute promyelocytic leukemia with all-trans retinoic acid and anthracycline monochemotherapy: long-term outcome of the LPA 99 multicenter study by the PETHEMA Group. Blood. 2008;112(8):3130-4.

8. Kim YJ, Cho SG, Lee $S$, et al. Potential role of adoptively transferred allogeneic WT1-specific CD4+ and CD8+ T lymphocytes for the sustained remission of refractory AML. Bone Marrow Transplant. 2010;45(3):597-9.

9. Tsuboi A, Oka Y, Kyo T, et al. Long-term WT1 peptide vaccination for patients with acute myeloid leukemia with minimal residual disease. Leukemia. 2012;26(6):1410-3.
Submit your next manuscript to BioMed Central and we will help you at every step:

- We accept pre-submission inquiries

- Our selector tool helps you to find the most relevant journal

- We provide round the clock customer support

- Convenient online submission

- Thorough peer review

- Inclusion in PubMed and all major indexing services

- Maximum visibility for your research

Submit your manuscript at www.biomedcentral.com/submit
Biomed Central 